\title{
A woman with low HDL cholesterol and corneal opacity
}

\author{
Tiziano Lucchi $\cdot$ Laura Calabresi $\cdot$ Angela Pinto \\ Elisa Benetti • Beatrice Arosio - Sara Simonelli • \\ Roberto Ratiglia $\cdot$ Carlo Vergani
}

Received: 8 June 2011/Accepted: 13 October 2011/Published online: 29 October 2011

(C) SIMI 2011

\section{Case presentation}

Dr. Lucchi

A 50-year-old woman was referred to our metabolic diseases clinic for high triglycerides and low HDL cholesterol in the blood. She also had corneal opacity. There was no family history of dyslipidemia or cardiovascular disease. She recalled that blood triglycerides had increased on taking hormone medication for metrorrhagia, but had normalised after stopping treatment. The corneal opacity had developed in adolescence, with low-light vision deficit and photophobia with snow glare. She reported mild anaemia which was attributed to the metrorrhagia, and for which she took periodic oral iron supplement. The rest of her history was unremarkable.

Her weight was $57 \mathrm{~kg}$, BMI $21.5 \mathrm{~kg} / \mathrm{m}^{2}$, and waist circumference $74 \mathrm{~cm}$; bilateral corneal opacity and corneal arcus were plainly evident (Fig. 1); tendinous xanthomas and xanthelasmas were absent; the tonsils were normal;

T. Lucchi $(\square) \cdot$ E. Benetti $\cdot$ B. Arosio $~ C$. Vergani Metabolic Diseases Clinic,

Geriatric Operating Unit, Fondazione IRCCS Cà Granda Ospedale Maggiore Policlinico, University of Milan, Milan, Italy e-mail: tlucchi@policlinico.mi.it

L. Calabresi · S. Simonelli

Centro Grossi Paoletti, Dipartimento of Pharmacological

Science, University of Milan, Milan, Italy

A. Pinto $\cdot$ R. Ratiglia

Ophthalmology Operating Unit,

Fondazione IRCCS Cà Granda Ospedale Maggiore Policlinico,

University of Milan, Milan, Italy cardiorespiratory, abdominal and neurological examinations were normal.

Table 1 shows some blood and urine parameters. Total cholesterol, LDL cholesterol and particularly HDL cholesterol were low. The cholesterol was largely non-esterified. Apo A-I, Apo B, Apo C-II, and Apo C-III were also low. Triglycerides were high. There was mild macrocytic normochromic anaemia, with stomatocytes on a peripheral blood smear. Transaminases were slightly high; markers for hepatitis B and C were absent. Total protein, iron, vitamin B12, folate, homocysteine, and indicators of thyroid and renal function were all within normal limits. Urinalysis showed no microalbuminuria.

Doppler ultrasound of the supra-aortic vessels was unremarkable (IMT $\max 0.75 \mathrm{~mm}$ ); however, it incidentally revealed thyroid nodules suggesting multi-nodular normo-functioning goitre. Abdominal ultrasound revealed hyperreflective aortic walls due to calcium deposition, but was negative for liver and kidney disease. Exercise stress testing showed no evidence of reduced coronary reserve.

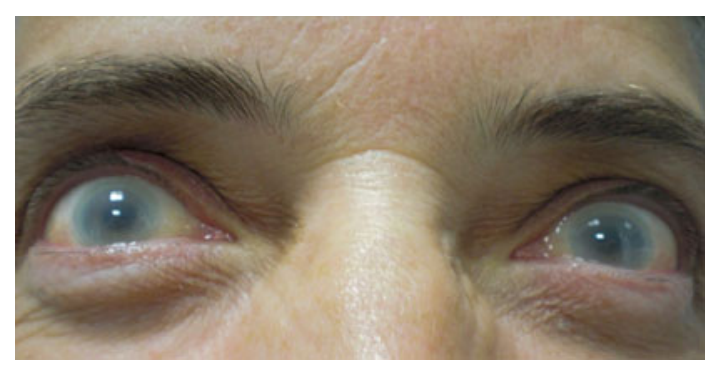

Fig. 1 Photograph of the patient showing corneal opacity and corneal arcus 
Table 1 Blood and urine parameters of the patient

\begin{tabular}{lll}
\hline Variable & Reference value & Patient value \\
\hline Total cholesterol (mg/dl) & $<200$ & 155 \\
Esterified cholesterol (\% of total) & $>70$ & $<20$ \\
LDL cholesterol (mg/dl) & $<130$ & 89 \\
HDL cholesterol (mg/dl) & $>50$ & 8 \\
Triglycerides (mg/dl) & $<170$ & 287 \\
Apolipoprotein A-I (mg/dl) & $108-225$ & 38 \\
Apolipoprotein B (mg/dl) & $60-133$ & 39 \\
Apo B/Apo A-I & $<0.8$ & 1.02 \\
Apolipoprotein C-II (mg/dl) & $1.6-4.2$ & 0.2 \\
Apolipoprotein C-III (mg/dl) & $5.5-9.5$ & 4.4 \\
Lipoprotein(a) (mg/dl) & $<30$ & $<6$ \\
Haemoglobin (g/dl) & $12-16$ & 11.8 \\
Mean corpuscular volume (fl) & $78-99$ & 101 \\
Aspartate aminotransferase (U/l) & $5-32$ & 35 \\
Alanine aminotransferase (U/l) & $5-31$ & 58 \\
Gamma glutamyltransferase (U/l) & $5-36$ & 58 \\
Creatinine (mg/dl) & $0.5-1$ & 0.98 \\
Urea (mg/dl) & $15-50$ & 37 \\
Glucose (mg/dl) & $70-110$ & 85 \\
Microalbuminuria (mg/l) & $<20$ & 8.6 \\
\hline
\end{tabular}

Table 2 Primary dyslipoproteinemias associated with low HDL cholesterol

Analphalipoproteinemia (Tangier disease)

Familial hypoalphalipoproteinemia

Cholesteryl ester storage disease (CESD)

Familial hypertriglyceridemia

Familial lecithin: cholesterol acyltransferase (LCAT) deficiency

\section{Differential diagnosis}

\section{Dr. Lucchi, Dr. Benetti}

In making the differential diagnosis we considered primary (Table 2) and secondary (Table 3) dyslipoproteinemias associated with low HDL cholesterol.

In Tangier disease, HDL cholesterol is almost completely absent and the levels of Apo A-I are 1-3\% of normal; blood triglycerides may be high and stomatocytes may be present in the peripheral blood. At a clinical examination, the tonsils appear yellow-orange and increased in size; there is hepatosplenomegaly and peripheral neuropathy. The cornea may be diffusely or focally opaque.

In familial hypoalphalipoproteinemia, HDL cholesterol levels are below the 10th percentile of the normal range, apolipoprotein A-I levels are about $50 \%$ of normal, and blood triglycerides may be high. There is a family history of myocardial infarction and early death.
Table 3 Conditions and drugs associated with low HDL cholesterol

Obstructive liver disease (hepatitis)

Chronic intestinal disease with malabsorption

Dysthyroidism

Infection

Lymphoma (myeloproliferative disease)

Collagenopathies

Cystic fibrosis

Chronic anaemia

Diabetes mellitus

Acute myocardial infarction

Chronic renal failure

Nephritic syndrome

Malnutrition

Alcohol abuse

Obesity

Drugs: $\beta$-blockers, thiazide diuretics, estrogens, progestins, androgens, glucocorticoids, isotretinoin, valproate, bile acid sequestrants, cyclosporine, protease inhibitors, benzodiazepines, sulfonylureas

In cholesteryl ester storage disease, HDL cholesterol is low and often associated with high blood triglycerides; total and LDL cholesterol are high. Hepatomegaly, hepatic steatosis and large artery atheromas are also present.

In familial hypertriglyceridemia, HDL cholesterol is low and triglycerides are high with an inverse hyperbolic correlation between the two. Hepatic steatosis is often present.

Familial lecithin-cholesterol acyltransferase (LCAT) deficiency is characterised by low HDL cholesterol and low Apo A-I; total cholesterol and triglycerides may be normal or high, while esterified cholesterol levels are markedly reduced. The main clinical manifestations are corneal opacity, nephropathy and anaemia.

\section{Further investigations}

\section{Prof. Ratiglia, Dr. Pinto}

Examination by slit biomicroscopy revealed diffuse opacity of the corneal stroma, peripherally more prominent, in both eyes. A $360^{\circ}$ peripheral corneal arcus was present that was somewhat indistinct at the peripheral margins and towards the limbal border.

The anterior chambers were deep and unremarkable. The lenses were correctly located and transparent.

Intra-ocular pressure was $14 \mathrm{mmHg}$ and visus was $8 / 10$ in both eyes. Right eye correction was $-0.25 \mathrm{SPH},-0.25$ CYL axis at $70^{\circ}$; left eye correction was $-1.25 \mathrm{SPH},-0.75$ CYL axis at $20^{\circ}$; the prescription for near vision was +2.00 $\mathrm{SPH}$. 
Fundus examination indicated normal posterior pole and peripheral retina confirmed by Heidelberg retinal angiography. Contrast sensitivity, tested with Pelli Robson chart at $1 \mathrm{~m}$ and the patient emmetropic, was below normal. Computerised visual field testing (central 30-2 threshold test) revealed diffuse mild-to-moderate reduction of retinal sensitivity. Heidelberg retinal tomography (Rostock Cornea Module) showed a normal corneal epithelium but the corneal sub-basal nerve plexus was poorly discernible. However, Cochet-Bonnet esthesiometry indicated normal corneal sensitivity $(58 \mathrm{~mm})$.

The corneal stroma was altered throughout its thickness and extent, with poorly recognisable keratocytes, and widely distributed patches of irregular hyperreflectivity, interrupted by multiple hyporeflective striations. Some rounded hyperreflective patches tended to fuse with each other and appeared to be accumulations of extracellular cholesterol, while the hyporeflective areas were the interface between collagen fibres and cholesterol deposits.

Corneal pachymetry (Pentacam) revealed corneal thickness nearly double the normal, greater at the periphery $(1,067 \mu \mathrm{m}$ right; $1,115 \mu \mathrm{m}$ left) than apex (reference $530 \mu \mathrm{m})$. Astigmatism was regular $\left(1.4 \mathrm{D}\right.$ at $\left.71.8^{\circ}\right)$ in the right eye and oblique $\left(2.1 \mathrm{D}\right.$ at $\left.134^{\circ}\right)$ in the left eye.

Ocular US showed a normal eyeball with no retinal detachments, and a thickened sclera; there were no signs of vitreous disease and no masses.

Ultrabiomicroscopy (Fig. 2) confirmed the corneal thickening (most marked peripherally close to the limbus) and showed a deep anterior chamber without iridocorneal adhesions or masses.

\section{Prof. Calabresi, Dr. Arosio}

The clinical history and examination as well as the lipoprotein profile were strongly suggestive of LCAT deficiency. We therefore assessed plasma LCAT which was found to be $5.1 \mu \mathrm{g} / \mathrm{ml}$ (normal range 3.1-6.7 $\mu \mathrm{g} / \mathrm{ml}$ ). The esterification of cholesterol within endogenous lipoproteins [cholesterol esterification rate (CER)] or incorporated into an exogenous standardised substrate (LCAT activity) were both zero.

Sequencing of the LCAT gene showed a $\mathrm{C}$ to $\mathrm{T}$ substitution at c.511 (c.511C $\rightarrow \mathrm{T})$ of exon 4 of both alleles, resulting in the substitution of tryptophan for arginine at position 147 of the protein (R147W).

The proband's sons (aged 29 and 30) were also analysed. The levels of total cholesterol and triglycerides were normal or near-normal, but HDL cholesterol was low (Table 4). LCAT activity and CER were somewhat below normal. Each son had inherited a single allele of the mother's mutated gene.

\section{Diagnosis}

The patient is affected by familial LCAT deficiency due to a single mutation $(\mathrm{c} .511 \mathrm{C} \rightarrow \mathrm{T})$ in both alleles of the LCAT gene resulting in an almost complete loss of enzyme activity.

\section{Discussion}

Prof. Vergani, Dr. Lucchi

LCAT is a glycoprotein enzyme, 416 amino acids long, which is produced in the liver and released in the plasma mainly in association with HDL particles. It catalyses the transfer of a polyunsaturated fatty acid residue from position 2 of lecithin to the hydroxyl group (position 3) of the free cholesterol present in nascent discoidal HDL particles,
Fig. 2 Ultrabiomicroscopy. The arrows indicate thickening of the peripheral cornea close to the limbus
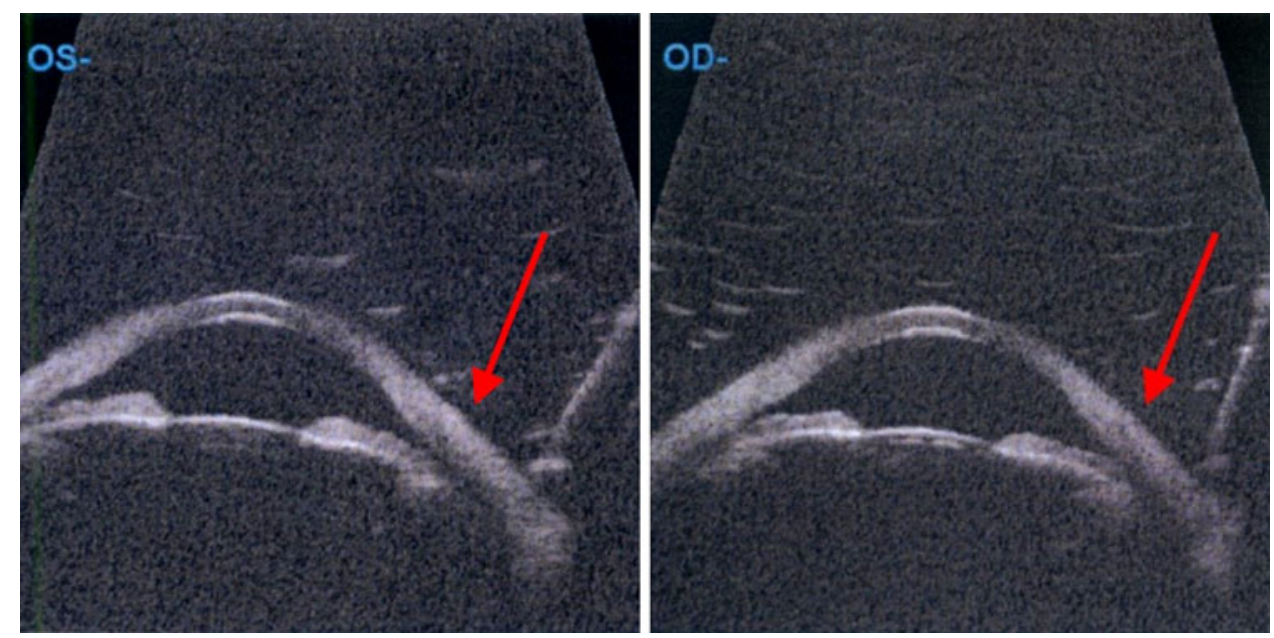
Table 4 Lipoprotein profiles and LCAT levels and activity in the two sons of the proband

\begin{tabular}{llll}
\hline Variable & Reference value & 30 -year-old son & 29-year-old son \\
\hline Total cholesterol (mg/dl) & $<200$ & 186 & 205 \\
LDL cholesterol (mg/dl) & $<130$ & 133 & 154 \\
HDL cholesterol (mg/dl) & $>50$ & 29 & 38 \\
Triglycerides $(\mathrm{mg} / \mathrm{dl})$ & $<170$ & 116 & 90 \\
Apolipoprotein A-I (mg/dl) & $104-202$ & 102 & 107 \\
Apolipoprotein B (mg/dl) & $60-133$ & 100 & 104 \\
Apolipoprotein C-II (mg/dl) & $1.6-4.2$ & 4.3 & 3.8 \\
Apolipoprotein C-III $(\mathrm{mg} / \mathrm{dl})$ & $5.5-9.5$ & 8.6 & 9.2 \\
Lipoprotein(a) $(\mathrm{mg} / \mathrm{dl})$ & $0-30$ & 6 & 16 \\
Apo B/Apo A-I & $<0.8$ & 0.98 & 0.97 \\
LCAT ( $\mu$ g/ml) & $3.1-6.7$ & 4.0 & 4.7 \\
LCAT activity $(\mathrm{nmol} / \mathrm{ml} / \mathrm{h})$ & $25-55$ & 18.9 & 11.0 \\
CER $(\mathrm{nmol} / \mathrm{ml} / \mathrm{h})$ & $30-60$ & 21.3 & 20.5 \\
\hline
\end{tabular}

producing lysolecithin and cholesteryl ester. Apolipoprotein A-I, a structural protein of HDLs, is a cofactor for this reaction. The enzyme therefore converts free cholesterol into a non-polar compound that migrates into the hydrophobic core of the HDL particles, enabling them to achieve their mature spherical shape $[1,2]$.

The LCAT gene is located on chromosome 16q22. Over 40 mutations of this gene have been described, resulting in a reduced enzyme activity and two main clinical phenotypes: familial LCAT deficiency and fish-eye disease [1, 3-5]. In familial LCAT deficiency, LCAT is absent or non-functional, CER is zero, almost all plasma cholesterol is non-esterified, HDLs are virtually absent and those present are discoidal in shape like nascent HDLs. In addition, foam cells containing cholesterol and phospholipids accumulate in various tissues typically in cornea, kidney, liver, spleen and bone marrow. The main clinical manifestations are corneal opacity, nephropathy with albuminuria, and anaemia with target cells; some patients also develop early atherosclerosis $[1,3]$.

Fish-eye disease is a condition in which LCAT is unable to esterify HDL cholesterol, but is capable of esterifying the cholesterol of those lipoproteins that contain Apo B. As a result, plasma levels of esterified cholesterol are not so drastically reduced as in LCAT deficiency $[1,3]$.

The present case of familial LCAT deficiency was characterised by a corneal opacity that compromised visual acuity only moderately, although the overall quality of vision was quite markedly compromised, as revealed by the visual contrast and visual field investigations, and the patient's complaints of difficulties in low-light conditions and snow glare. Visual acuity in familial LCAT deficiency is known to vary [6-9].

Our patient also suffered from mild macrocytic normochromic anaemia, even though plasma levels of iron, vitamin B12 and folate were normal. It is recognised that in familial LCAT deficiency erythrocytes may have a shorter half-life because their altered plasma membrane composition entails morphological abnormalities and an increased fragility that may favour splenic sequestration $[1,2,10]$. The modest increase in serum transaminases found in our patient, uncommon in this condition, is probably due to the accumulation of foam cells and lipid vesicles in the liver in the absence of hepatomegaly [1].

It is noteworthy that there were no signs of altered renal function in our patient. This was also the case in an Italian family with the same LCAT mutation [3] whose members exhibited clinical features like our patient; corneal opacity and anaemia but no renal compromise. Instead in the patient in whom this mutation R147W has been first reported [11], all the distinctive clinical features of the disease were present. It has been shown that the phenotype of familial LCAT deficiency is influenced by the nature of the mutation as well as by post-transcriptional factors that modify LCAT activity [3, 12, 13].

It should be noted that the very low HDL levels in our patient had never been evaluated before she was referred to our metabolic diseases clinic. Over the years, she had been prescribed repeated blood tests for total cholesterol and triglycerides. The former had always been low, and she had been told this meant protection from atherosclerotic disease. The triglycerides had occasionally been found to be high, and interpreted as a consequence of the hormone therapy she was taking. We believe our case report prompts a reflection on the widespread clinical practice of using total cholesterol as a screening tool, and HDL cholesterol as a second-line test to be performed only if total cholesterol is found to be high. As far as cardiovascular risk is concerned, a few points deserve mention. Although it is acknowledged that low levels of HDL cholesterol lead to an increased risk of cardiovascular disease [14], familial LCAT deficiency does not seem to be invariably associated 
with greater cardiovascular morbidity or mortality $[2,15]$. In the case of our patient, there was no family history of early cardiovascular events, and Doppler ultrasound and exercise stress testing excluded signs of major atherosclerosis. Thus, her cardiovascular risk did not appear to be increased even though she had very low levels of HDL cholesterol, a high Apo B/Apo A-I ratio and carried variant II of the I405V polymorphism of the cholesteryl ester transfer protein, all factors which are known to be associated with a greater likelihood of cardiovascular disease [16-18]. Conversely, an 18-year-old patient with familial LCAT deficiency previously described by us had a family history of early myocardial infarction and sudden death [19]. The role of LCAT in the protection against atherosclerosis thus remains a controversial issue[20-22].

There is no effective treatment for familial LCAT deficiency [1]. In order to keep blood triglyceride levels under control, patients are advised to follow a low-fat diet, and to take ethyl esters of omega-3 fatty acids. Dialysis may become necessary if severe renal failure develops. With regard to the corneal opacity, corneal transplant is indicated in the case of loss of sight, and may also be required in the presence of cataracts to allow visualisation of the lens structure during surgery [7-9]. It must, however, be noted that no long-term follow-up data are available in the literature, and that cholesterol build-up may recur in the transplanted flap. Another possible ocular manifestation is the rupture of Bruch's membrane that has sometimes been described in familial LCAT deficiency, and can be complicated by choroid neovascularisation resulting in severe visual deficits [7] and retinal haemorrhage. The follow-up of these patients should therefore include an assessment of both the anterior and posterior segments of the eye.

Conflict of interest None.

\section{References}

1. Santamarina-Fojo S, Hoef J, Assmann G, Brewer HB Jr (2001) Lecithin: cholesterol acyltransferase deficiency and fish-eye disease. In: Scriver CR, Beaudet A, Valle D, Sly WS (eds) The metabolic and molecular bases of inherited disease, 8th edn. McGraw Hill, New York, pp 2817-2834

2. Jonas A (2000) Lecithin cholesterol acyltransferase. Biochim Biophys Acta 1529:245-256

3. Calabresi L, Pisciotta L, Costantin A et al (2005) The molecular basis of lecithin: cholesterol acyltransferase deficiency syndromes. A comprehensive study of molecular and biochemical findings in 13 unrelated Italian families. Arterioscler Thromb Vasc Biol 25:1972-1978

4. Norum KR, Gjone E (1967) Familial serum-cholesterol failure: a new inborn error of metabolism. Biochim Biophys Acta 144:698700
5. Carlson LA, Philipson B (1979) Fish-eye disease. A new familial condition with massive corneal opacities and dyslipoproteinaemia. Lancet 2:922-924

6. Viestenz A, Schlotzer-Schrehardt U, Hofmann-Rummelt $\mathrm{C}$ et al (2002) Histopathology of corneal changes in lecithin-cholesterol acyltransferase deficiency. Cornea 21:834-837

7. Hirano K, Kachi S, Ushida C, Naito M (2004) Corneal and macular manifestations in a case of deficient lecithin: cholesterol acyltransferase. Jpn J Ophthalmol 48:82-84

8. Palmiero PM, Sbeity Z, Liebmann J et al (2009) In vivo imaging of the cornea in a patient with lecithin-cholesterol acyltransferase deficiency. Cornea 28:1061-1064

9. Winder AF, Owen JS, Pritchard PH et al (1999) A first British case of fish-eye disease presenting at age 75 years: a double heterozygote for defined and new mutations affecting LCAT structure and expression. J Clin Pathol 52:228-230

10. Suda T, Akamatsu A, Nakaya Y et al (2002) Alterations in erythrocyte membrane lipid and its fragility in a patient with familial lecithin: cholesterol acyl transferase (LCAT) deficiency. J Med Invest 49:147-155

11. Taramelli R, Pontoglio M, Candiani G et al (1990) Lecithin cholesterol acyl transferase deficiency: molecular analysis of a mutated allele. Hum Genet 85:195-199

12. Kosman J, Jonas A (2001) Deletion of specific glycan chains affects differentially the stability, local structures, and activity of lecithin-cholesterol acyltransferase. J Biol Chem 276:3723037236

13. Kuivenhoven JA, Pritchard H, Hill J et al (1997) The molecular pathology of lecithin: cholesterol acyltransferase (LCAT) deficiency syndromes. J Lipid Res 38:191-205

14. Gordon DJ, Probstfield JL, Garrison RJ et al (1989) High-density lipoprotein cholesterol and cardiovascular disease. Four prospective American studies. Circulation 79:8-15

15. Ayyobi AF, McGladdery SH, Chan S et al (2004) Lecithin: cholesterol acyltransferase (LCAT) deficiency and risk of vascular disease: 25-year follow-up. Atherosclerosis 177:361-366

16. McQueen MJ, Hawken S, Wang X et al (2008) Lipids, lipoproteins and apolipoproteins as risk markers of myocardial infarction in 52 countries (the INTERHEART study): a case-control study. Lancet 372:224-233

17. Vergani C, Lucchi T, Caloni M et al (2006) I405V polymorphism of the cholesteryl ester transfer protein (CETP) gene in young and very old people. Arch Gerontol Geriatr 43:213-221

18. Thompson A, Di Angelantonio E, Sarwar N et al (2008) Association of cholesterol ester transfer protein genotypes with CETP mass and activity, lipid levels, and coronary risk. JAMA 299: 2777-2788

19. Vergani C, Catapano AL, Roma P, Giudici G (1983) A new case of familial LCAT deficiency. Acta Med Scand 214:173-176

20. Calabresi L, Baldassarre D, Castelnuovo S et al (2009) Functional lecithin: cholesterol acyltransferase is not required for efficient atheroprotection in humans. Circulation 120:628-635

21. Rousset X, Vaisman B, Amar M et al (2009) Lecithin: cholesterol acyltransferase-from biochemistry to role in cardiovascular disease. Curr Opin Endocrinol Diabetes Obes 16:163-171

22. Sethi AA, Sampson M, Warnick R et al (2010) High pre-beta1 HDL concentrations and low lecithin: cholesterol acyltransferase activities are strong positive risk markers for ischemic heart disease and independent of HDL-cholesterol. Clin Chem 56:1128-1137 